\title{
Impact of strain effects on the stability of Ising walls in ferroelectrics
}

\author{
P. V. Yudin, ${ }^{*}$ A. K. Tagantsev, and N. Setter \\ Ceramics Laboratory, Swiss Federal Institute of \\ Technology (EPFL), CH-1015 Lausanne, Switzerland
}

(Dated: December 4, 2013)

\begin{abstract}
The internal structure of neutral 180-degree domain walls in Perovskite-type ferroelectrics with tetragonal symmetry of ferroelectric phase is analytically studied taking into account electromechanical coupling. It is shown that the widely used approximation neglecting the elastic effects may lead to qualitatively wrong results. Specifically, we address structural transitions from Ising (achiral) to Bloch (chiral) state in $180^{\circ}$ domain walls. In the approximation neglecting strain effects, stability of Ising walls is determined by the competition of correlation and Landau energies; domain walls are expected to be chiral near the boundaries between two different ordered phases, where polarization rotation is not costly. We demonstrate that such reasoning does not hold when elastic effects are taken into account. The stability condition is severely affected by the elastic effects. Sufficient condition for the stability of Ising walls may be formulated only in terms of elastic and electrostrictive properties, regardless the correlation energy. We demonstrate the results by considering tetragonal PZT. It is shown that elasticity stabilizes Ising walls and makes chiral walls unfavorable for any composition of the material, while nonelastic model predicts chirality of 180-degree walls near the morphotropic boundary.
\end{abstract}




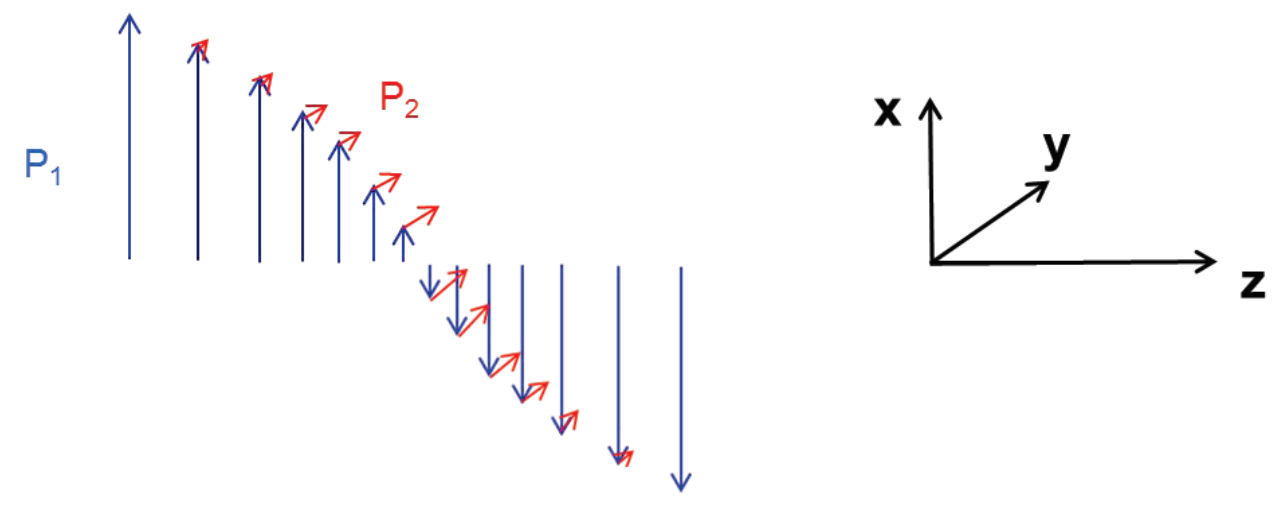

FIG. 1. The polarization vector distribution in space

\section{INTRODUCTION}

With advances of nanotechnology, domain walls start being considered as individual objects rather than just boundaries separating two domains. Despite small thicknesses typically of several nanometers, domain walls can not only be detected, but also manipulated with the use of modern scanning probe microscopes. ${ }^{1}$. Perovskites present a large class of ferroelectric materials widely used in modern applications. The cubic symmetry of Landau potential allows perovskite domain walls to have variable internal structures. In our work we consider two types of 180-degree walls, or two phases of a wall, as we consider also transitions between these types. The first type is Ising-walls in which only one polarisation component $P_{1}$ is present $\left(P_{1}\right.$ in Fig. 1$)$. In the other type, additional polarization component $P_{2}$ arises, such walls are refered to as Bloch-walls ${ }^{2}$. The polarization profile in a Bloch wall may be obtained by summation of $P_{2}$ and $P_{1}$ in Fig. 1 , the resulting vector draws a helix on passing from one domain to the other. Bloch walls may represent left spirals as well as right spirals. The ground state of a Bloch wall is two-times energy-degenerate, that makes it bistable. This bistability could be of interest for nano-electronic applications. For example dense memory can be created on a ferroelectric sample with bistable 180-degree domain walls by writing sub-domains as illustrated in Fig. 2. It is essential to be able to switch the wall from one state to another, hence, the regions on the phase-diagram of the wall near transition from Ising type to Bloch-bistable type are attractive for study, due to the small energy barrier for switching there. Question of stability of Ising walls was studied theoreticaly in the frame of simple model neglecting electromechanical coupling ${ }^{3}$. On 
the basis of this theory a conclusion may be drawn that at the vicinity of the boundary of two different ordered phases (morphotropic boundary) any 180-degree domain wall becomes chiral. This can be understood as following. The tendency of a wall to be in one phase or the other may be explained through interaction between the two order parameter components. The competition of $P_{1}$ and $P_{2}$ makes the rise of $P_{2}$ in the bulk of the domains unfavorable. In the domain wall, where $P_{1}$ passes through zero, there is a prerequisite for $P_{2}$ to rise, although the gradient term acts against its appearance. Thus depending on the relative ratio of the gradient and Landau energies, a wall may be of Bloch- or of Ising-type. Because in the vicinity of a morphotropic boundary Landau energy weakly increases from polarisation rotation, domain walls are expected to become thick and chiral. Never the less, the chiral walls were not yet observed in Tetragonal phase. Numerical calculations based on Ginsburg-Landau-Devonshire theory with account of electromechanical coupling ${ }^{4}$, does not reveal Bloch walls in the tetragonal phase. In this work we develop analytical model to reconsider the question of stability of Ising walls taking into account electro-mechanical coupling. In the model we include electrostrictive interaction and neglect flexoelectric effect. We demonstrate the results on the parameters of $\mathrm{Pb}\left(\mathrm{Zr}_{1-c} T i_{c}\right) O_{3}$ (PZT). The influence of

the flexoelectricity on the domain wall structure in tetragonal ferroelectrics is studied in ${ }^{5}$, from where it follows that the effect is expected to be small in PZT with high Ti-fraction. We show that elastic effects introduce additional limitations for the appearance of chirality. This limitations are severe and a situation is possible in which for certain combination of elastic and electrostrictive properties of material, chiral walls are impossible for any values of the correlation energy terms.

\section{GIBBS POTENTIAL AND GOVERNING EQUATIONS}

We base our calculations on the Gibbs free energy density. In this section we start from potential expanded up to fourth power in terms of polarization. This model is valid for ferroelectrics with 2-nd order phase transition not too far from the transition temperature. Otherwise taking into account 6th power of polarization in the Landau expression is essential. We consider the changes introduced by addition of 6th power in section $\mathrm{V}$

$$
G=\frac{1}{2} A_{i j} P_{i} P_{j}+\frac{1}{4} B_{i j k l} P_{i} P_{j} P_{k} P_{l}+\frac{1}{2} D_{i j k l} \frac{\partial P_{i}}{\partial x_{j}} \frac{\partial P_{k}}{\partial x_{l}}-Q_{i j k l} \sigma_{i j} P_{k} P_{l}-\frac{1}{2} s_{i j k l} \sigma_{i j} \sigma_{k l}
$$




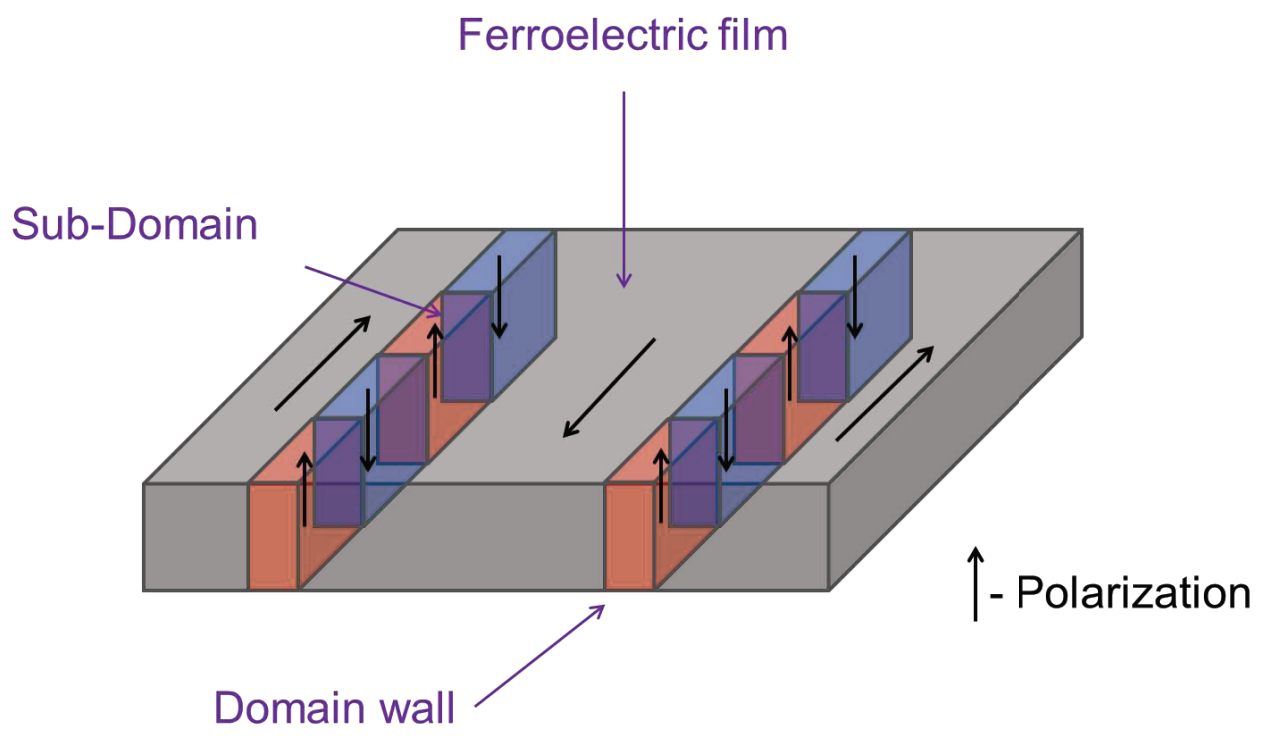

FIG. 2. Sub-domains in ferroelectric sample

where $A_{i j}=\alpha \delta_{i j}$ and $B_{i j k l}=\frac{\beta_{2}}{3}\left(\delta_{i j} \delta_{k l}+\delta_{i k} \delta_{j l}+\delta_{i l} \delta_{j k}\right)+\left(\beta_{1}-\beta_{2}\right) g_{i j k l}$, are the coefficients of Landau expansion, $\alpha$ being temperature dependent $\alpha=\alpha_{0}\left(T-T_{0}\right)$, and $\beta_{1}, \beta_{2}>0$;

$$
D_{i j k l}=D_{12} \delta_{i j} \delta_{k l}+D_{44}\left(\delta_{i k} \delta_{j l}+\delta_{i l} \delta_{j k}\right)+\left(D_{11}-D_{12}-2 D_{44}\right) g_{i j k l}
$$

is the gradient term. $\sigma_{i j}$ are the components of mechanical stress. Hereafter the summation over repeating indices is implied, $\delta_{i j}$ is invariant Kronecker tensor and $g_{i j k l}$ is the cubic tensor of 4th rank, having the following structure in crystallographic axes: $g_{i j k l}=1$ if $i=j=k=l$ and $g_{i j k l}=0$ otherwise.

Electrostriction and compliance tensors $Q_{i j k l}$ and $s_{i j k l}$ has same structures as $D_{i j k l}$ as a consequence of cubic symmetry.

$$
\begin{gathered}
Q_{i j k l}=Q_{12} \delta_{i j} \delta_{k l}+Q_{44}\left(\delta_{i k} \delta_{j l}+\delta_{i l} \delta_{j k}\right)+\left(Q_{11}-Q_{12}-2 Q_{44}\right) g_{i j k l} \\
s_{i j k l}=s_{12} \delta_{i j} \delta_{k l}+s_{44}\left(\delta_{i k} \delta_{j l}+\delta_{i l} \delta_{j k}\right)+\left(s_{11}-s_{12}-2 s_{44}\right) g_{i j k l}
\end{gathered}
$$

From the Gibbs potential one obtains equations of state

$$
\frac{\partial G}{\partial P_{i}}-\frac{d}{d x}\left(\frac{\partial G}{\partial P_{i}^{\prime}}\right)=0
$$


Besides we imply condition of mechanical equilibrium

$$
\frac{\partial \sigma_{i j}}{\partial x_{j}}=0(i, j=1,2,3)
$$

and Poisson's equation

$$
\frac{d\left(\varepsilon_{b} E_{i}+P_{i}\right)}{d x_{i}}=0
$$

Where $\varepsilon_{b}$ is the background dielectric permittivity, $E_{i}$ is the vector of electric field, and $P_{i}$ is the ferroelectric part of the polarization vector (hereafter we use the term polarization as shorthand).

Tetragonal phase with spontaneous polarization $P_{0 T}=\sqrt{\frac{-\alpha}{\beta_{1}}}$ and six equivalent domain states $\left\{P_{0 T}, 0,0\right\},\left\{-P_{0 T}, 0,0\right\},\left\{0, P_{0 T}, 0\right\}$,etc. is given by homogeneous solution of the system of equations (5),(6) at zero stress within $\alpha<0$ and $\beta_{2}>\beta_{1}$. Transition to paraelectric phase corresponds to $\alpha<0$; transition to the rhombohedral phase occurs at the morphotropic boundary, determined by $\beta_{2}=\beta_{1}$.

The planar 180-degree domain walls we are interested in are simplest connections between two oppositely poled domains. We consider one-dimensional problem with polarization vector $\mathbf{P}$ and mechanical stresses $\sigma_{i j}$ being dependent only on the coordinate $\mathbf{z}$ normal to the plane of the wall. Correspondingly, the boundary conditions are the following:

$$
\mathbf{P}=\mathbf{P}_{\mathbf{0 T}}, \sigma_{i j}=0 \text { for } x=-\infty \text { and } \mathbf{P}=\mathbf{P}_{\mathbf{0 T}}, \sigma_{i j}=0 \text { for } x=\infty i, j=1,2,3
$$

Here $\mathbf{P}_{\mathbf{0}}$ is the vector of spontaneous polarization in the domain $\mathrm{x} \rightarrow \infty$.

\section{APPROXIMATION IN THE ABSENCE OF ELECTROMECHANICAL COU- PLING}

As a benchmark, it is instructive to consider the problem without taking into account elastic effects, i. e. with $Q_{i j k l}$ and $s_{i j k l}$ tensors set to zero in (1). As we will show in the next section, where we consider the impact of electro-mechanical coupling, the addition of non-zero $Q_{i j k l}$ and $s_{i j k l}$ lead to a result with same mathematical structure, but with renormalized coefficients in the Landau expansion. For ferroelectrics with a large value of the dielectric permittivity with respect to background permittivity (7) may be rewritten 


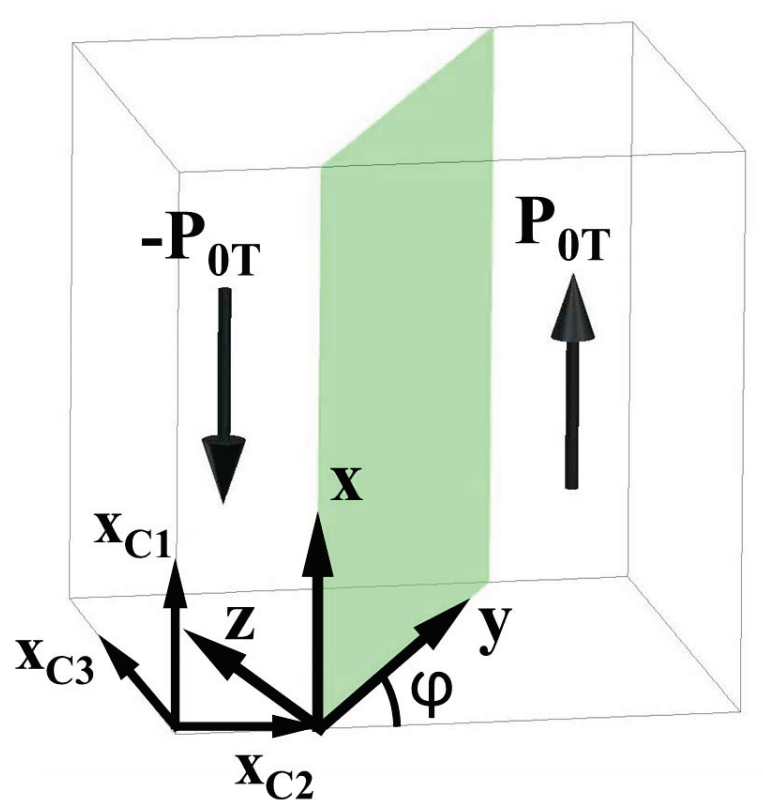

FIG. 3. Wall orientations and related coordinate set. $\varphi$ is the dihedral angle between the wall plane and the (001) plane.

as $\operatorname{div} \mathbf{P}=0$ or $P_{z}=0$ in the one-dimensional case. The orientations of the domain walls compatible with this condition with respect to crystallographic axes $x_{C 1} x_{C 2} x_{C 3}$ are shown in Fig. 3. We introduce the right-handed coordinate set related to the wall with $\mathrm{x}$-axis directed along the vector of spontaneous polarization and $\mathrm{y}$ orthogonal to $\mathrm{x}$ and $\mathrm{z}$ as shown in the figure. In what follows we use notations $P_{1}$ and $P_{2}$ for $\mathrm{x}$ - and y-polarization components correspondingly.

With the aforementioned simplifications, The Gibbs potential (1) in the reference frame related to a domain wall, takes the form:

$$
G_{T}=\frac{1}{2} \alpha\left(P_{1}^{2}+P_{2}^{2}\right)+\frac{1}{4} \beta_{1} P_{1}^{4}+\frac{1}{2} \beta_{2} P_{1}^{2} P_{2}^{2}+\frac{1}{4} \beta_{T}\left(P_{2}^{4}\right)+\frac{1}{2} \delta_{1}\left(\frac{d P_{1}}{d x}\right)^{2}+\frac{1}{2} \delta_{2}\left(\frac{d P_{2}}{d x}\right)^{2}
$$

where $\beta_{T}=\left(3 \beta_{1}+\beta_{2}+\left(\beta_{1}-\beta_{2}\right) \cos 4 \varphi\right) / 4, \delta_{1}=D_{44}$ and $\delta_{2}=\delta_{1}\left(\frac{1+\cos 4 \varphi}{2}+\frac{1-\cos 4 \varphi}{2 \Delta}\right), \Delta=$ $\frac{2 D_{44}}{D_{11}-D_{12}}$ is the gradient term anisotropy parameter.

From (9) one obtains equations of state 


$$
\begin{gathered}
\alpha P_{1}+\beta_{1} P_{1}^{3}+\beta_{2} P_{1} P_{2}^{2}-\delta_{1} \frac{\partial^{2} P_{1}}{\partial z^{2}}=0 \\
\alpha P_{2}+\beta_{T} P_{2}^{3}+\beta_{2} P_{2} P_{1}^{2}-\delta_{2} \frac{\partial^{2} P_{2}}{\partial z^{2}}=0
\end{gathered}
$$

The Ising wall is the solution of equations (10) with only one non-zero polarization component $P_{1}$. Its profile is given by the well-known formula ${ }^{6}$, same for all compatible wall orientations.

$$
P_{1}=P_{0 T} \tanh (z / t)
$$

where $t=\sqrt{-2 \delta_{1} / \alpha}$ has a meaning of the domain wall half-width.

However, this solution does not always describe a stable 180-degree wall. If it is not stable with respect to appearance of the additional polarization component, it means that Bloch wall solution becomes energetically favorable. One can note that if the polarization component profiles $P_{1}(z), P_{2}(z)$ satisfy the system $(10)$, then $P_{1}(z),-P_{2}(z)$ will also be a solution, and this two states are energetically equivalent as (9) does not contain odd powers of $P_{2}$. It signifies bistability of the Bloch walls. There is no analytical solution available for the developed Bloch-walls. We limit our analysis to indicating conditions when Ising wall looses stability, that can be obtained analytically. The limit of stability of the Ising solution can be found using the technique developed for this kind of problems ${ }^{7}$. We look for the minimum eigenvalue of the functional acting on $P_{2}$, obtained from Eq. (10b) where for $P_{1}(x)$ we put the solution (11) and linearize it with respect to $P_{2}$. If it is negative then the Ising solution is unstable. One readily finds this functional in the form

$$
\left(-\frac{\partial^{2}}{\partial x^{2}}+B+U \tanh ^{2}\left(\frac{z}{t}\right)\right) P_{2}
$$

where $B=\alpha / \delta_{2} ; U=\beta_{2} P_{0 T}^{2} / \delta_{2}$. Its smallest eigenvalue can be found in the form.

$$
\left(\sqrt{4 U t^{2}+1}-1+2 B t^{2}\right) / 2 t^{2}
$$

The stability condition may be written as:

$$
\frac{\beta_{2}}{\beta_{1}}>1+2 \frac{\delta_{1}}{\delta_{2}} \text { or } \frac{\beta_{2}}{\beta_{1}}>1+\frac{4 \Delta}{1+\Delta+(\Delta-1) \cos (4 \varphi)}
$$




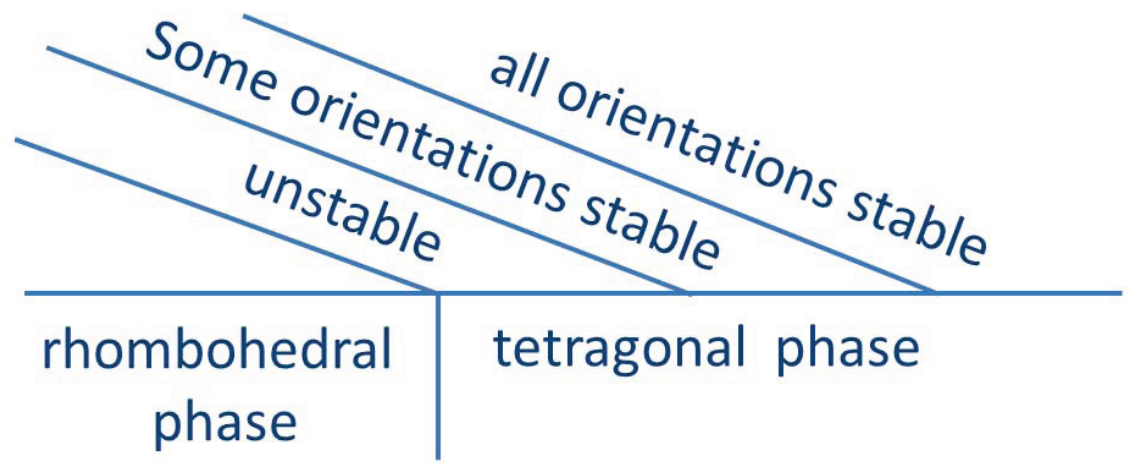

FIG. 4. Stability Chart for the Ising wall profile in the approximation in the absence of electromechanical coupling. Parameter $\beta_{2} / \beta_{1}$ increases from the left to the right

The equality in (14) corresponds to a phase transition, when a new component arises in the wall. According to the relationship (14) near the morphotropic boundary the domain walls are chiral for any orientations. The stability chart interpreting (14) as a function of parameter $\beta_{2} / \beta_{1}$ is illustrated in Fig. 4. Since the parameter $\frac{\delta_{1}}{\delta_{2}}$ depends on the wall orientation, a region on the chart may exist where some walls are of Ising type, others - of Bloch type.

\section{ACCOUNT OF ELASTIC EFFECTS}

In this section we describe the mechanical state of the sample, and indicate the way the problem is mathematically reduced to one considered in the previous section.

The strains that raise in the sample can be found from the constitutive equations of elasticity:

$$
\varepsilon_{i j}=-\frac{\partial G}{\partial \sigma_{i j}}
$$

Far from the domain wall we apply the conditions of a stress-free sample:

$$
\sigma_{i j}\left(x_{3} \rightarrow \pm \infty\right)=0 ; i, j=1,2,3
$$

From (15) (at $\mathbf{P}=\mathbf{P}_{\mathbf{0 T}}, \sigma_{i j}=0$ ) one obtains boundary conditions for the strain components:

$$
\varepsilon_{11}=\varepsilon_{11}^{0} ; \varepsilon_{22}=\varepsilon_{22}^{0} ; \varepsilon_{12}=0
$$


where

$$
\varepsilon_{11}^{0}=Q_{11} P_{0 T}^{2} ; \quad \varepsilon_{22}^{0}=Q_{12} P_{0 T}^{2} ;
$$

Mechanical equilibrium conditions (6) could be written as $\partial \sigma_{3} / \partial x_{3}=0, \partial \sigma_{4} / \partial x_{3}=0$, $\partial \sigma_{5} / \partial x_{3}=0$. Taking into account (16) one obtains $\sigma_{3}=\sigma_{4}=\sigma_{5}=0$ everywhere. In the case of $z$-dependent solution, the Saint-Venant compatibility relations

$$
e_{i k l} e_{j m n}\left(\partial^{2} \varepsilon_{l n} / \partial x_{k} \partial x_{m}\right)=0
$$

where $e_{i j k}$ is the Levi-Civita symbol, transform to

$$
d^{2} \varepsilon_{11} / d x_{3}^{2}=d^{2} \varepsilon_{12} / d x_{3}^{2}=d^{2} \varepsilon_{22} / d x_{3}^{2}=0
$$

The solution to Eq. (20) with boundary conditions (16) is:

$$
\varepsilon_{1}(z)=\varepsilon_{1}^{0} ; \varepsilon_{2}(z)=\varepsilon_{2}^{0} ; \varepsilon_{6}(z)=0
$$

Note that it is the only possible one-dimensional solution for the elastic problem. The applicability of this solution to a stress free sample with finite sizes is equivalent to the applicability of a one-dimensional model to a parallel plate capacitor. By applying this we neglect the fringe elastic fields at the contact of the domain wall with the surface, which is permissible when the dimensions of the sample are much larger than the thickness of the domain wall.

Eqs. (21) , (15) yield:

$$
\begin{array}{r}
s_{11} \sigma_{11}+s_{12} \sigma_{22}+Q_{11} P_{1}^{2}+Q_{12} P_{2}^{2}=Q_{11} P_{0 T}^{2} \\
\frac{1}{4}\left(4 s_{12} \sigma_{11}+3 s_{11} \sigma_{22}+s_{12} \sigma_{22}+2 s_{44} \sigma_{22}+\left(s_{11}-s_{12}-2 s_{44}\right) \sigma_{22} \cos 4 \varphi+\right. \\
\left.+4 Q_{12} P_{1}^{2}+\left(3 Q_{11}+Q_{12}+2 Q_{44}+\left(Q_{11}-Q_{12}-2 Q_{44}\right) \cos 4 \varphi\right) P_{2}^{2}\right)=Q_{12} P_{0 T}^{2} \\
4\left(s_{44} \sigma_{6}+Q_{44} P_{1} P_{2}\right)=0
\end{array}
$$

From (22) we obtain expressions for the nonzero elastic stress components in the form: 


$$
\begin{array}{r}
\sigma_{11} \equiv \sigma_{1}=\frac{\left(P_{0 T}^{2}-P_{1}^{2}\right)\left(Q_{11} s_{22}(\varphi)-Q_{12} s_{12}\right)+P_{2}^{2}\left(Q_{22}(\varphi) s_{12}-Q_{12} s_{22}(\varphi)\right)}{s_{22}(\varphi) s_{11}-s_{12}^{2}} \\
\sigma_{22} \equiv \sigma_{2}=\frac{\left(Q_{12} s_{11}-Q_{11} s_{12}\right)\left(P_{0 T}^{2}-P_{1}^{2}\right)+P_{2}^{2}\left(-s_{11} Q_{22}(\varphi)+Q_{12} s_{12}\right)}{s_{22}(\varphi) s_{11}-s_{12}^{2}} \\
\sigma_{12} \equiv \sigma_{6}=-\frac{Q_{44}}{s_{44}} P_{1} P_{2} \\
s_{22}(\varphi)=s_{11}+\sin ^{2}(2 \varphi)\left(\frac{s_{44}}{4}-\frac{s_{11}-s_{12}}{2}\right) \\
Q_{22}(\varphi)=Q_{11}+\sin ^{2}(2 \varphi)\left(\frac{Q_{44}}{4}-\frac{Q_{11}-Q_{12}}{2}\right)
\end{array}
$$

Euler Eqs. (5) with elastic effects taken into account read as:

$$
\begin{array}{r}
\alpha P_{1}+\beta_{1} P_{1}^{3}+\beta_{2} P_{1} P_{2}^{2}-2\left(Q_{11} \sigma_{11} P_{1}+Q_{12} \sigma_{22} P_{1}+2 Q_{44} \sigma_{12} P_{2}\right)-\delta_{1} \frac{\partial^{2} P_{1}}{\partial z^{2}}=0 \\
\alpha P_{2}+\beta_{T} P_{2}^{3}+\beta_{2} P_{2} P_{1}^{2}+\frac{1}{4}\left(-16 Q_{44} \sigma_{12} P_{1}-8 Q_{12} \sigma_{11} P_{2}-\right. \\
\left.-2 \sigma_{22}\left(3 Q_{11}+Q_{12}+2 Q_{44}+\left(Q_{11}-Q_{12}-2 Q_{44}\right) \cos 4 \varphi\right) P_{2}\right)-\delta_{2} \frac{\partial^{2} P_{2}}{\partial z^{2}}=0
\end{array}
$$

Substitution of (23) into (24) yields:

$$
\begin{array}{r}
\alpha^{\prime} P_{1}+\beta_{1}^{\prime} P_{1}^{3}+\beta_{2}^{\prime} P_{1} P_{2}^{2}-\delta_{1} \frac{\partial^{2} P_{1}}{\partial z^{2}}=0 \\
\alpha^{\prime \prime} P_{2}+\beta_{1}^{\prime \prime} P_{2}^{3}+\beta_{2}^{\prime} P_{2} P_{1}^{2}-\delta_{2} \frac{\partial^{2} P_{2}}{\partial z^{2}}=0
\end{array}
$$

where following renormalization is done:

$$
\alpha^{\prime}=\alpha-P_{0 T}^{2} \Theta ; \alpha^{\prime \prime}=\alpha+\Omega P_{0 T}^{2} ; \beta_{1}^{\prime}=\beta_{1}+\Theta ; \beta_{2}^{\prime}=\beta_{2}+\Xi
$$

which is equivalent to the following using relation $P_{0 T}=\frac{-\alpha}{\beta_{1}}$ :

$$
\alpha^{\prime}=\alpha\left(1+\frac{\Theta}{\beta_{1}}\right) ; \alpha^{\prime \prime}=\alpha\left(1-\frac{\Omega}{\beta_{1}}\right) ; \beta_{1}^{\prime}=\beta_{1}\left(1+\frac{\Theta}{\beta_{1}}\right) ; \beta_{2}^{\prime}=\beta_{2}+\Xi
$$

where 


$$
\begin{array}{r}
\Theta=\frac{Q_{11}^{2}\left(s_{11}-s_{12}-\frac{s_{44}}{2}\right) \cos (4 \alpha)+Q_{11}^{2}\left(3 s_{11}+s_{12}+\frac{s_{44}}{2}\right)-8 Q_{12} Q_{11} s_{12}+4 Q_{12}^{2} s_{11}}{\left(s_{11}-s_{12}-\frac{s_{44}}{2}\right) s_{11} \cos (4 \alpha)+3 s_{11}^{2}+\left(s_{12}+\frac{s_{44}}{2}\right) s_{11}-4 s_{12}^{2}}(28 \mathrm{a}) \\
\Omega=\left(2 Q_{11}^{2} s_{12}(\cos (4 \alpha)+3)+(28 \mathrm{~b})\right. \\
+Q_{12}\left(Q_{44} s_{11}(\cos (4 \alpha)-1)+2 Q_{12}\left(s_{11}(\cos (4 \alpha)-1)+4 s_{12}\right)\right)- \\
\left.-Q_{11}\left(Q_{44} s_{12}(\cos (4 \alpha)-1)+2 Q_{12}\left(s_{44} \sin ^{2}(2 \alpha)+2 s_{11}(\cos (4 \alpha)+3)\right)\right)\right) \cdot \\
\cdot\left(s_{11}\left(2 s_{12}+s_{44}\right) \sin ^{2}(2 \alpha)+s_{11}^{2}(\cos (4 \alpha)+3)-4 s_{12}^{2}\right)^{-1} \\
\Xi=\frac{Q_{44}^{2}}{s_{44}}-\Omega(28 \mathrm{c})
\end{array}
$$

Thus the equations has the same structure as in the approximation neglecting elastic effects, but now $\alpha$ depend on the wall orientation and is different for the two Euler equations. This enriches the variety of possible cases for the stability condition. In particular, if $\alpha^{\prime \prime}$ becomes positive, we have "paraelectric phase" for the second component of polarization. It means that the appearance of the second polarization component is not favorable even in the middle of the domain wall, where $P_{1}=0$, regardless the value of correlation terms. In this case we say that Ising solution is "safely" stable. The complementary case can be formulated as requirement (necessary condition) for the bistablity in the wall:

$$
\beta_{1}-\Omega>0
$$

If condition (29) is met it means that appearance of $P_{2}$ in the middle of the domain wall decreases the polarization-related energy density. Although the gradient term may still suppress this new component. To determine whether the single-component solution is stable we use the same method as in section III. Taking into account elastic effects, by analogy to (12), (13),(14) we obtain stability condition for Ising walls:

$$
\frac{\beta_{2}^{\prime}}{\beta_{1}^{\prime}}>\frac{\alpha^{\prime \prime}}{\alpha^{\prime}}\left(1+2 \frac{\delta_{1} \alpha^{\prime \prime}}{\delta_{2} \alpha^{\prime}}\right)
$$

which transforms to the following with account of renormalizations (27)

$$
\frac{\beta_{2}+\Xi}{\beta_{1}-\Omega}>1+2 \frac{\beta_{1}-\Omega}{\beta_{1}+\Theta} \frac{\delta_{1}}{\delta_{2}}
$$




\section{GENERALIZATION OF THE THEORY FOR FERROELECTRICS WITH $1^{\text {st }}$ ORDER PHASE TRANSITION}

The Gibbs potential $G_{1}$ for ferroelectrics with 1-st order phase transition may be obtained from $\mathrm{G}(1)$ as following:

$$
G_{1}=G+C_{i j k l m n} P_{i} P_{j} P_{k} P_{l} P_{m} P_{n}
$$

where $C_{i j k l m n}=\frac{\gamma_{3}}{6}<\delta_{i j} \delta_{k l} \delta_{m n}>+\left(\gamma_{2}-\frac{\gamma_{3}}{2}\right)<\delta_{i j} g_{k l m n}>+\left(\gamma_{1}-\gamma_{2}+\frac{\gamma_{3}}{3}\right) g_{i j k l m n}^{(6)}$ is the $6^{\text {th }}$ order dielectric stiffness tensor. With $<>$ we denote symmetrization with respect to interchange of indices: e.g. $\left\langle\delta_{i j} \delta_{k l}>=\frac{1}{3}\left(\delta_{i j} \delta_{k l}+\delta_{i k} \delta_{j l}+\delta_{i l} \delta_{j k}\right), g_{i j k l m n}^{(6)}\right.$ is invariant tensor of $6^{\text {th }}$ rang for the cubic symmetry. In the cubic crystallographic axes the tensor $g_{i j k l m n}^{(6)}=1$ if $i=j=k=l=m=n$ and $g_{i j k l m n}^{(6)}=0$ otherwise.

Minimisation of $G_{1}$ yields the following expression for the spontaneous polarization in the domains ${ }^{6}$ :

$$
P_{s}=\sqrt{\frac{\sqrt{\beta_{1}^{2}-24 \gamma_{1} \alpha}-\beta_{1}}{12 \gamma_{1}}}
$$

One can check that the elastic renormalizations due to electrostriction derived in section IV do not involve the $6^{\text {th }}$ order dielectric stiffness tensor $C_{i j k l m n}$. Thus the condition (29) may be reformulated for $1{ }^{s t}$ order ferroelectrics just by change $P_{O T}$ to $P_{s}$ in expression (26). Thus we can formulate requirement (necessary condition) for the appearence of bistablity in the wall in first order ferroelectrics as follows:

$$
\frac{12 \gamma_{1} \alpha}{\sqrt{\beta_{1}^{2}-24 \gamma_{1} \alpha}-\beta_{1}}+\Omega<0
$$

Note that this expression should be applied also for the materials with 2-nd order phase transition in the temperature range far from the transition point where the influence of 6th polarisation power becomes essential. There is no possibility to derive exact analytical expression analogous to (30), which would take into account the correlation energy. However, from the structure of Eqs. (25) it follows that the stability of Ising walls is governed by the same factor $\delta_{1} / \delta_{2}$. In the next section we explore stability of Ising domain walls for the 
TABLE I. Values of the thermodynamical parameters of PZT at $25^{\circ} \mathrm{C}$

(* -values obtained by extrapolation)

\begin{tabular}{|l|l|l|l|l|l|l|l|l|}
\hline Parameter $\backslash \mathrm{c}$ & 0.4 & 0.5 & 0.6 & 0.7 & 0.8 & 0.9 & 1.0 & Refs. \\
\hline$\alpha\left(10^{8} \mathrm{~m} / F\right)$ & -1.58 & -0.98 & -1.67 & -2.49 & -2.97 & -3.24 & -3.42 & 8 \\
\hline$\beta_{1}\left(10^{8} \mathrm{~m}^{5} / C^{2} F\right)$ & 6.65 & 1.91 & 1.45 & 0.26 & -1.22 & -2.32 & -2.91 & 8 \\
\hline$\beta_{2}\left(10^{8} \mathrm{~m}^{5} / C^{2} F\right)$ & 4.78 & 3.47 & 6.47 & 10.2 & 12.6 & 14.1 & 15 & 8 \\
\hline$\gamma_{1}\left(10^{8} \mathrm{~m}^{9} / C^{4} F\right)$ & 2.71 & 1.33 & 1.86 & 2.35 & 2.47 & 2.52 & 2.60 & 8 \\
\hline$\gamma_{2}\left(10^{8} \mathrm{~m}^{9} / C^{4} F\right)$ & 12.1 & 6.13 & 8.50 & 10.25 & 9.68 & 8.01 & 6.10 & 8 \\
\hline$\gamma_{3}\left(10^{8} \mathrm{~m}^{9} / C^{4} F\right)$ & -5.69 & -2.89 & -4.06 & -5.00 & -4.90 & -4.36 & -3.66 & 8 \\
\hline$Q_{11}\left(10^{-2} \mathrm{~m}^{4} / C^{2}\right)$ & 7.26 & 9.66 & 8.12 & 7.89 & 8.14 & 8.50 & 8.90 & 8 \\
\hline$Q_{12}\left(10^{-2} \mathrm{~m}^{4} / C^{2}\right)$ & -2.71 & -4.60 & -2.95 & -2.48 & -2.45 & -2.5 & -2.6 & 8 \\
\hline$Q_{44}\left(10^{-2} \mathrm{~m}^{4} / C^{2}\right)$ & 6.29 & 8.19 & 6.71 & 6.36 & 6.42 & 6.57 & 6.75 & 8 \\
\hline$s_{11}\left(10^{-12} \mathrm{~Pa}^{-1}\right)$ & 8.8 & 10.5 & 8.6 & 8.4 & 8.2 & 8.1 & $8.0^{*}$ & 9 \\
\hline$s_{11}\left(10^{-12} \mathrm{~Pa}^{-1}\right)$ & -2.9 & -3.7 & -2.8 & -2.7 & -2.6 & -2.5 & $-2.4^{*}$ & 9 \\
\hline$s_{11}\left(10^{-12} \mathrm{~Pa}^{-1}\right)$ & 24.6 & 28.7 & 21.2 & 17.5 & 14.4 & 12 & $10^{*}$ & 9 \\
\hline
\end{tabular}

parameters of $\mathrm{Pb}\left(\mathrm{ZrO}_{1-c} \mathrm{Ti}_{c}\right) \mathrm{O}_{3}$ (PZT). Depending on the Ti-fraction c the phase transition may be of 2 nd or 1 st order. In case of 2 nd order we use analitical expressions, for the 1st order we solve the problem numerically.

\section{ANALYSIS OF STABILITY OF ISING DOMAIN WALLS IN PZT}

The solid solution $\mathrm{Pb}\left(\mathrm{ZrO}_{1-c} \mathrm{Ti}_{c}\right) \mathrm{O}_{3}$ has tetragonal structure if c is larger than 0.43 (morphotropic boundary). The cubic-tetragonal phase transition is of the 2-nd order if $c \leq 0.7$ and 1-st order for $c>0.7$. The thermodynamic coefficients for PZT for $\mathrm{c}$ values $0.4 ; 0.5 ; 0.6 ; 0.7 ; 0.8 ; 0.9 ; 1$ are listed in Table 1 . For other $\mathrm{c}$ in the interval we interpolate the parameters by polynomials.

To adress the question of stability of Ising walls we first check inequality (34). The lefthand part of the condition (34) is illustrated in Fig. 5 as a function of Ti-concentration. It is seen that for $c<0.62$ Ising walls are "safely" stable for any wall orientation. Thus 


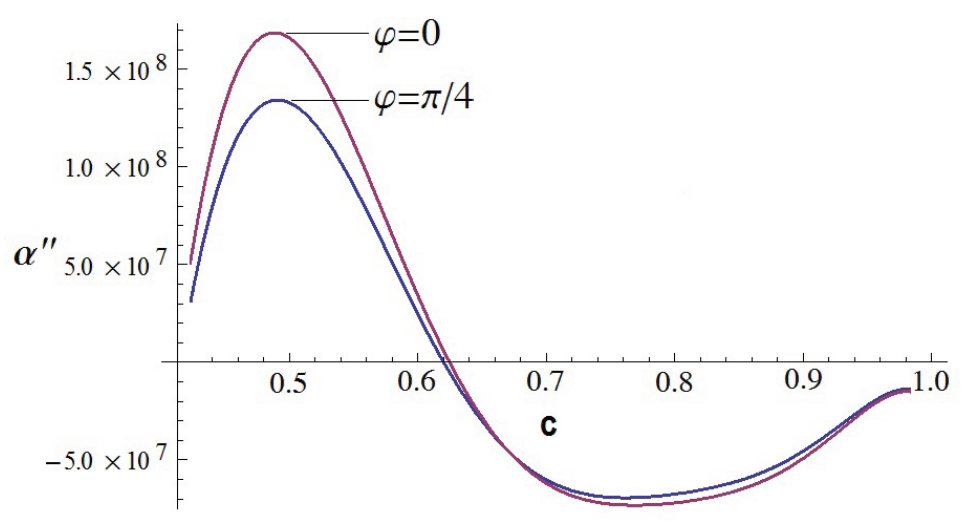

FIG. 5. Dependance of the factor $\alpha^{\prime \prime}=\frac{12 \gamma_{1} \alpha}{\sqrt{\beta_{1}^{2}-24 \gamma_{1} \alpha}-\beta_{1}}+\Omega$ on the Ti-fraction $\mathrm{c}$ in PZT

the statement that all the domain walls near the morphotrophic boundry become chiral, that was obtained in the "not elastic" model, does not hold for the case when the elastic effects are taken into account. In the region where $\alpha^{\prime \prime}<0$ the stability of Ising walls is determined by the factor $\delta_{1} / \delta_{2}$. In material with tetragonal symmetry $\delta_{1} / \delta_{2}$ varies in the interval $(1, \Delta)$, where $\Delta=\frac{2 D_{44}}{D_{11}-D_{12}}$ is the gradient term anisotropy parameter; this follows from expression (14). Wall orientation $\varphi=0$ corresponds to $\delta_{1} / \delta_{2}=1 ; \delta_{1} / \delta_{2}=\Delta$ at $\varphi=\pi / 4$. We consider the extreme case $\varphi=\pi / 4$, and we vary $\Delta$-parameter since it is unknown for PZT. Domain wall structure is calculated numerically for different $\Delta$-values for $c=0.7$, see Fig. 6 . The case $\Delta \rightarrow \infty$ demonstrates the value of $P_{2}$-component conditioned by elastic effects only. Note that in "not elastic" approximation the maximal value of $P_{2}$ would be equal to unity. Thus we observe the suppression of the $P_{2}$-component by elastic effects only. Switching on gradually the correlation term by decreasing $\Delta$ we observe further suppression and smoothing of the hump and it completely vanishes at $\Delta \approx 40$. From the numerical calculations we see that chiral walls are favourable only under extremely high $\delta_{1} / \delta_{2}$ - values, that are not realistic for PZT (for pure $\mathrm{PbTiO}_{3} \delta_{1} / \delta_{2} \approx 1.7^{10}$ ).

\section{CONCLUSION}

Taking into account the electromechanical coupling leads to qualitatively new results for the stability of Ising walls in perovskite ferroelectrics. Elastic renormalisations may lead to "paraelectric phase" for the 2-nd polarization component, implying "safe" stability of Ising walls, regardless the correlation energy anisotropy. This "paraelectric phase" occurs in 


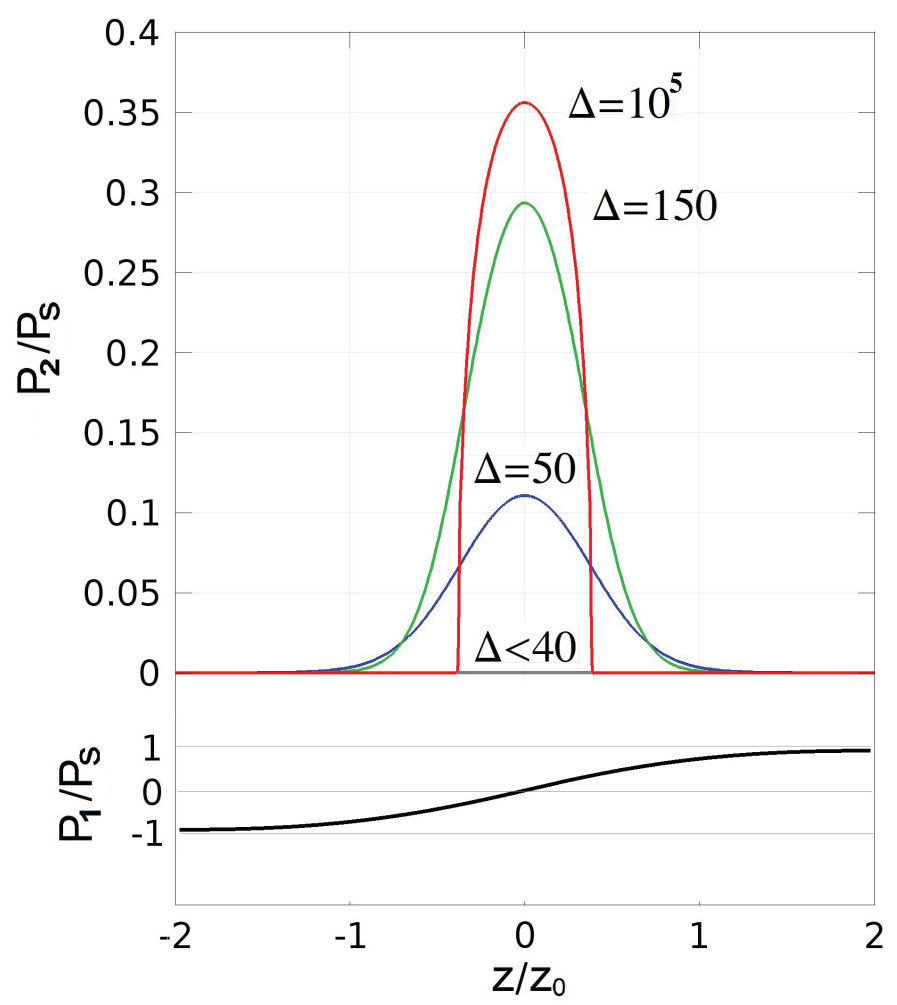

FIG. 6. Dependence of domain wall profile on the parameter $\Delta$.

tetragonal PZT close to the morphotropic boundary, which makes chiral walls impossible. Thus the conclusion of the "not elastic" theory that all the domain walls near the morphotrophic boundry become chiral does not hold for the case when the elastic effects are taken into account.

\section{ACKNOWLEDGEMENT}

This publication is based on work supported by EU project ERC-268058 MOBILE-W.

* petr.yudin@epfl.ch

1 V. Aravind, A. N. Morozovska, S. Bhattacharyya, D. Lee, S. Jesse, I. G. Y. Li, S. Choudhury, P. Wu, K. Seal, A. Rappe, S. Svechnikov, E. Eliseev, S. Phillpot, L. Chen, V. Gopalan, and S. Kalinin, Physical Review B. 82, 024111 (2010).

2 V. Stepkova, P. Marton, and J. Hlinka, J. Phys.: Condens. Matter 24, 212201 (2012). 
3 B. Houchmandzadeh, J. Lajzerowicz, and E. Salje, Journal of Physics-Condensed Matter 3, $5163(1991)$.

4 P. Marton, I. Rychetsky, and J. Hlinka, Physical Review B 81, 144125 (2010).

5 P. V. Yudin, A. K. Tagantsev, E. A. Eliseev, A. N. Morozovska, and N. Setter, Physical Review B. 86, $134102(2012)$.

6 A. K. Tagantsev, L. E. Cross, and J. Fousek, "Domains in ferroic crystals and thin films," (Springer, New York, 2010) Chap. 6.2.2, pp. 300-304.

7 L. Bulaevskii and B. Vekhter, Soviet physics - JETP 64, 851 (1986).

8 M. J. Haun, E. Furman, S. Jang, and L. Cross, Ferroelectrics 99, 45 (1989).

9 N. A. Pertsev, V. G. Kukhar, H. Kohlstedt, and R. Waser, Physical Review B. 67, 054107 (2003).

10 M. Kempa, J. Hlinka, J. Kulda, P. Bourges, A. Kania, and J. Petzelt, Phase Transitions 79, 351 (2006), 17th Czech-Polish Seminar on Structural and Ferroelectric Phase Transitions MAY 22-26, 2006 Znojmo, CZECH REPUBLIC. 